Case Report

\title{
Variation in the origin of right obturator artery - A case report
}

\author{
Rani Nallathamby ${ }^{1}$, Ramakrishna Avadhani', Sivarama C.H. ${ }^{3}$, Meril Ann Soman ${ }^{4} \&$ Meera Jacob ${ }^{5}$ \\ ${ }^{1,3}$ Post Graduates, ${ }^{2}$ Professor \& HOD, ${ }^{4}$ Lecturer, ${ }^{5}$ Assistant Professor, \\ Department of Anatomy, Yenepoya M edical College, M angalore - 575 018, Karnataka, India.
}

\author{
Correspondence \\ Rani Nallathamby \\ Postgraduate, Department of Anatomy, Yenepoya Medical College, Deralakatte, Mangalore. \\ Mobile : t91 8861120119 E-mail : raniboban@yahoo.com
}

\begin{abstract}
M ost commonly, the Obturator artery arises from the anterior trunk of internal iliac artery. However, origin of the Obturator artery from external iliac artery was reported at 25\% by M issankov et al. [3], 1.1\% by Bergman et al. [1], 1.3\% by Jakubowicz and CzerniawskaGrzesinska [2].Due to its high frequency of variations in course and origin, Obturator artery had drawn the attention of anatomists, surgeons and radiologists. In this case report, we are presenting an anomalous origin of right Obturator artery from right external iliac artery. The knowledge of this variation is important anatomically, radiologically and surgically.
\end{abstract}

Keywords : Obturator artery, external iliac artery, pelvic vessel variations.

\section{Introduction}

The rapid development of surgical and investigatory techniques and procedures, especially in the field of urology, obstetrics and gynecology, makes the study of the vascular patterns and their variations in the pelvis much important [4].Normally, the Obturator artery inclines anteroinferiorly on the lateral pelvic wall to the upper part of Obturator foramen. In the pelvis, it is related laterally to Obturator fascia separating it from Obturatorinternus and is crossed medially by the ureter and the ductus deferens separating it from parietal peritoneum with Obturator nerve running above and the Obturator vein below the artery [5]. It supplies the muscles of the medial compartment of the thigh.The present case is an observation of unilateral variation in the origin of right obturator artery which deserves attention and is a finding of curiosity due to its clinical significance.

\section{Case Report}

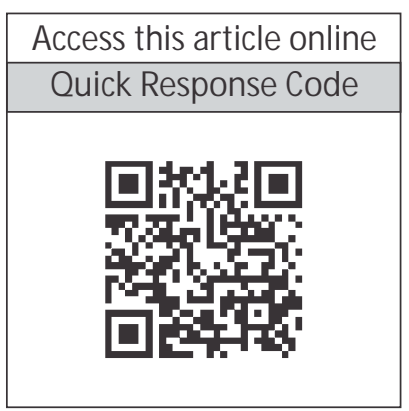

During routine dissection of $\quad \mathrm{medical}$ undergraduates in Yenepoya M edical College, Mangalore, Karnataka; it was noted that the Obturator artery on the right side of approximately
60 year old male cadaver, origins from right external iliac artery, passed medially, superficial to the external iliac vein, crossed the pelvic brim and descended anteriorly into the Obturator canal. The Obturator nerve and the vein entered the canal below the artery (Figure 1). The external iliac artery also had the other two normal branches namely the inferior epigastric artery and deep circumflex iliac artery. The Obturator vein drained normally into internal iliac vein. The vascular pattern in right side of pelvis appeared to be completely normal.

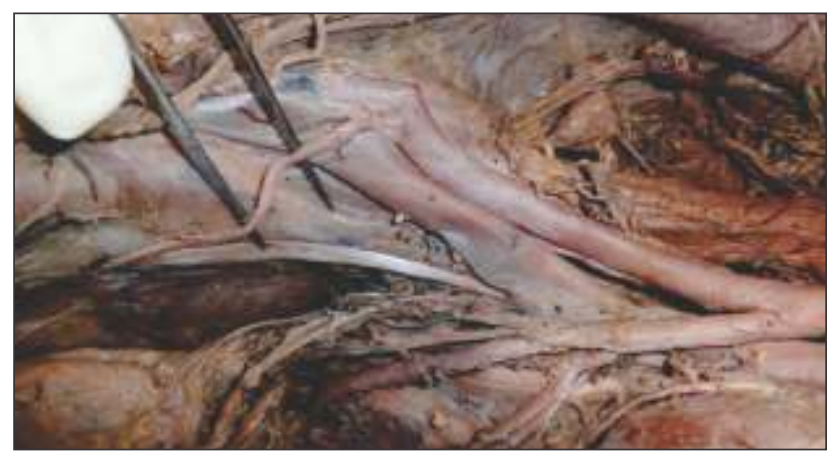

\section{Discussion}

The area of pelvic brim and lateral pelvic wall is very important and it is the anchoring site for inguinal and femoral hernia repairs and also for radical cystectomies. During the surgical procedure, the abdominal muscles are retracted laterally by applying pressure on the lateral pelvic wall (6). 
Bergman et al. [1] reported that the origin of the Obturator artery from the external iliac is not very common, and rarely occurs except in females. Similarly, our case was a male cadaver.Jakubowicz and Czerniawska-Grzesinska [2] studied variability in origin and topography of the inferior epigastric and Obturator arteries and found that in $4 \%$ of cases there was a common trunk for inferior epigastric and Obturator arteries. They also described that Obturator artery originated from inferior epigastric artery in $2.6 \%$ of their cases, and from external iliac artery in $1.3 \%$ of their cases.

The variation in the origin of Obturator artery can be due to its comparatively late development from the plexus which in turn is joined by the axial artery of lower limb that accompanies the sciatic nerve [7]. It is currently accepted that the anomalies affecting the arterial patterns of limbs are based on the unusual selection of channels from primary capillaries. The most appropriate channel enlarges, while others retract and disappear, thereby establishing a final arterial pattern and resulting in variations in origin.The origin of obturator artery from external iliac artery,as in this case, may be caused by such an unusual choice of channels. [8]

The variations in Obturator artery may lead to various complications during pelvic surgeries; mainly those require suturing along the pelvic brim. It may cause alarming hemorrhage if cut during hernia repairs or hip surgeries.In cases of ligation of the internal iliac arteries and their branches in women undergoing pelvic surgery, as well as in

\section{References}

1. Bergman R, Thompson SA, Afifi AK, Saadek FA (1988) Compendium of human anatomical variation. Urban Schwarzenberg, Baltimore, pp. 40-41.

2. Jakubowicz M, Czarniawska-Grzesinska M (1996) Variability in origin and topography of the inferior Epigastric and Obturator arteries. Folia Morphol, 55:121-126.

3. Missankov AA, Asvat R, Maoba KI (1996) Variations of the pubic vascular anastomoses in black South Africans.ActaAnat, 155: 212-214.

4. Chandler FA, Seidler F (1939) Intrapelvic Extra peritoneal Resection of cases of obstruction of the internal iliac artery due to any cause, the Obturator artery and its branches will be spared, especially the branch to the head of femur, when the Obturator artery arises from the external iliac artery. The relevance of this paper is to draw attention to those engaged in interventional maneuver into the human pelvis, as a variant Obturator vessel can be inadvertently cut and result in very serious complications, for example, during femoral ring procedures or laparoscopic interventions (2).The knowledge of the relations of the Obturator artery is important in intrapelvic extra peritoneal resection of the Obturator nerve in the treatment of spastic paralysis. Unexpected hemorrhage will occur if the artery- closely related to the nerve- is cut (4).It may lead to external iliac vein compression and venous stagnation of lowerlimb leading to complications.Tracing along the abnormal vessels can easily identify the Obturator foramen which is an anatomical landmark for inferior dissection of preperitonealspace.This case may be of special intrest to general and orthopedic surgeons.

\section{Conclusion}

The unexpected presence of the variant vessels in the pelvic region can become a matter of concern to orthopedic surgeons, urologists, gynecologists and general surgeons, who may perform various surgical procedures in this area as well as of academic interest to anatomists. The present case is an observation of unilateral variation in the origin of right Obturator artery which had emerged as a direct branch from external iliac artery. This is a very rare variation in Indian population.

The Obturator Nerve Surg. Gyn. Obs 69: 100-102.

5. Standring S (2005) Cardiovascular System in Gray's Anatomy Edinburg London New York Oxford Philadelphia St Louis Sydney Toronto 39: 1361

6. Gilroy AM, Hermey DC, DiBenedetto LM , M arks SC Jr, Page DW, Lei QF. Variability of the Obturator vessels. Clin Anat. 1997; 10: 328-332

7. Kumar D \&Rath G (2007) Anomalous Origin of Obturator artery from the internal iliac artery. Int. J. M orphol25: 639-641.

8. Arey LB (1954) the vascular system in Developmental Anatomy. WB Saunders Company 6: 364-377 